\title{
Weizmann Institute of Science
}

\section{Yoram Groner, Ph.D., Vice President, Professor of Molecular Genetics}

The Weizmann Institute of Science is a major center of scientific research and graduate study located on 300 landscaped acres in the town of Rehovot, Israel-14 miles south of Tel Aviv and 35 miles west of Jerusalem. The international campus community of 2,400 scientists and support staff includes more than 850 scientistsin-training at the Institute's Feinberg Graduate School.

The Institute's campus of about 70 buildings grew out of the modest Daniel Sieff Research Institute, founded in 1934 by a prominent Anglo-Jewish family, Israel and Rebecca Sieff, in memory of their son. The driving force behind its establishment was the Institute's first president, Dr. Chaim Weizmann, a noted chemist and statesman who later became the first president of Israel. On November 2, 1949, with the agreement of the Sieff family, the Institute was renamed and formally dedicated as the Weizmann Institute of Science.

In its early days, the Institute housed two main branches of research: organic chemistry and biochemistry. With a staff of ten accomplished scientists, Dr. Weizmann-who had already made significant contributions to organic chemistry and industrial fermentation-worked primarily in areas related to the region's fledgling economy: citrus industry, dairy farming, and pharmaceutical products.

At present, more than four decades after Dr. Weizmann's death, the Weizmann Institute is engaged in over 850 projects, both basic and applied, across the spectrum of contemporary research. Its 18 departments are grouped into five faculties: Biology, Biophysics-Biochemistry, Chemistry, Mathematical Sciences, and Physics. An additional department, Science Teaching, operates under the aegis of the Feinberg Graduate School.

The Institute's relatively small size and informal atmosphere are uniquely suited to the development of the interdisciplinary collaborations that increasingly characterize today's frontline science. For example, brain research at Weizmann brings together biologists, physicists, and mathematicians, whereas studies of the human genome, one of several areas in which Institute's scientists play a leading role nationally, involve biologists, biochemists, and computer scientists. To further enhance interdisciplinary interactions, the Institute has established 21 research centers, which are generally organized as intellectual rather than physical entities. These include centers for molecular genetics, neurosciences, higher brain functions, autoimmune diseases, the biology of aging, nutrition, plant biotechnology, and environmental research.

The Weizmann Institute has pioneered in various areas of science in Israel. It was the first to introduce cancer research locally, to establish a nuclear physics department and to have a research accelerator for the study of atomic nuclei. The first computer in Israel, and one of the first anywhere, was designed and built here. The Institute also houses the country's first, and so far only, submicron research facility for advancing tomorrow's electronics industry, as well as the first advanced solar energy research facility in Israel-one of only a handful worldwide. Through the Yeda Research and Development Company, which was established to further the commercialization of Weizmann Institute research, the Institute played a key role in the founding of Israel's first high-tech industrial park, Kiryat Weizmann, and of a new sciencebased industrial park now under construction near the campus. In recent years, an increasing number of research projects have successfully made the transition from lab to marketplace, both in Israel and abroad.

More than half of all Institute research involves in one way or another the study of the molecular biology of disease mechanisms. Weizmann scientists engage primarily in basic research, but many collaborate with physicians at other institutions in an effort to improve the diagnosis and treatment of various disorders.

Cancer research at the Weizmann Institute encompasses studies in cell differentiation, growth regulation, genetics, molecular therapeutics, and diagnostic approaches. The Institute's 


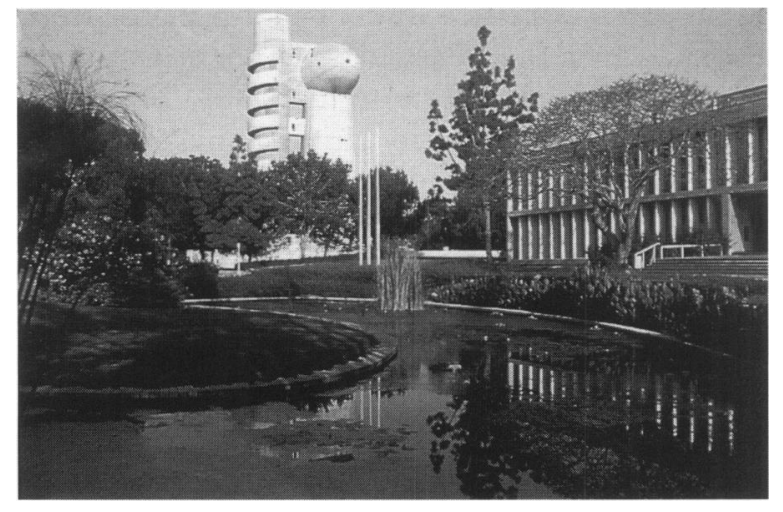

FIG. 1. The Weizmann Institute of Science, Rehovot, Israel

historic achievements include the identification of the genetic origins of some types of leukemia and the isolation of genes that induce or suppress malignancies, including $p 53$. Institute scientists produced the first monoclonal antibodies against $p 53$, providing a crucial tool for studying this gene's function and leading to the discovery that in cancer cells, normal $p 53$ undergoes a structural transformation and becomes dysfunctional. The currently accepted paradigm of cancer as a multistep process originated at the Institute, where researchers first recognized that at least two stages-initiation and promotion-are involved in tumor development.

Major efforts are directed at the study of autoimmune diseases, and two substances that were developed for the treatment of multiple sclerosis are already reaching patients. Other substances developed at the Institute are providing protection against hepatitis $\mathrm{B}$ and helping to treat the bone decalcification that accompanies kidney disease. Amniocentesis, now widely used to diagnose problems in the fetus, grew out of research originally conducted at the Institute, and a bone marrow transplant procedure developed by a biophysicist at the Institute has proven to be effective even in cases where there is no matched donor.

Other seminal contributions include the development of immobilized enzymes, which revolutionized several industries, including those dealing with the production of certain antibiotics. Weizmann studies have yielded important insights into the control of cell growth and differentiation, which helped to identify several families of proteins, including colony-stimulating factors and interleukins. Institute scientists also designed a remarkable biomolecular superglue, the avidin-biotin system, which has become the standard means of "gluing" together biological molecules and has given rise to a multimilliondollar market in areas ranging from purification of biochemicals to diagnosis of various diseases. A recent improvement in the superglue may significantly reduce the cost of many of its applications and lead to new uses, such as in the separation of cells for bone marrow transplants and in the isolation of DNA for genetic engineering.

Continuing studies are aimed at greater understanding of embryonic development, low birth weight, Down's syndrome, muscular dystrophy, and lissencephaly. In the area of brain research, currently a major focus of study at the Weizmann Institute, scientists have developed optical imaging, a new technique that allows the visualization of mental processes in real time at unprecedented resolution in time and space. Other studies deal with the dopamine and serotonin systems, the induction of nerve regeneration in the central nervous system, and the function of receptors suspected of involvement in Alzheimer's disease and amyotrophic lateral sclerosis.

These and other studies are primarily conducted in the Biology Faculty, which has recently been reorganized to create a more efficient environment for the evolving research in molecular biology. One of the newly formed departments is Biological Regulation, where researchers focus on the molecular signals that coordinate biochemical and cellular processes controlling the function of cells, tissues, and organs. Other new departments are Molecular Genetics and Molecular Cell Biology, whose scope includes the study of transcription regulation, protein degradation, cell death, and the mechanisms underlying organism development and morphogenesis.

Although molecular biology constitutes an important element of research, the Weizmann Institute also plays a leading role in other frontier areas of contemporary science. At the Institute's solar research facility, scientists and engineers are trying to develop new ways to harness the sun's energy. Scientists engaged in environmental studies analyze local aquifers and develop water protection and purification systems. Institute chemists work in areas ranging from the elucidation of such basic structures of life as the ribosome to the development of new materials. For example, the familiar photochromic materials that reversibly darken when exposed to sun- 
light are the result of a Weizmann Institute discovery.

Weizmann Institute plant geneticists have achieved dramatic improvements in the nutritional value of cultivated wheat and have made significant headway in working towards reducing the use of chemical fertilizers and herbicides. Taking a global view, a group at the Institute is assessing the effect of the damaged ozone layer on the process of photosynthesis.

Accomplishments of Institute mathematicians include the development of "smart cards" and decoders that prevent unauthorized access to confidential computer data and commercial satellite TV, and software architecture that allows people to meet "virtually" through the Internet. Institute software facilitates the development of sophisticated systems such as those used in aircraft, space shuttles, and nuclear power stations.

Institute physicists have made significant contributions to our understanding of the structure of elementary particles. They first proposed the existence of an elementary particle called the top quark and contributed to the identification of another particle called a gluon. They are currently playing an important role in the advanced experiments conducted at the European Laboratory for Particle Physics (CERN) in Geneva and at the DESY Laboratory in Hamburg. In the new field of submicron research, scientists are growing crystals in layers only a few atoms thick; this will result in smaller and faster computer chips for tomorrow's electronics industry.

The Institute's Feinberg Graduate School, which is both a recognized institution of higher learning in Israel and a school operating under charter from the Board of Regents of the State of New York, confers M.Sc. and Ph.D. degrees in the life sciences, chemistry, physics, mathematics, computer science, and science teaching. English is the official language of instruction and about $15 \%$ of the student body hails from abroad. Over the years, the Feinberg Graduate School has produced more than $30 \%$ of Israel's Ph.D.s in science and its alumni hold key positions both in Israel and overseas.

Consistent with the Institute's continuing preoccupation with the future is the work of the Science Teaching Department, which was established in 1968 under the aegis of the Feinberg Graduate School. This department continues to be devoted to the reform of science education in Israel. An energetic and imaginative Youth Activities section, developed for the encouragement of science-oriented youngsters, reaches out to more than 15,000 pupils annually with a variety of programs.

In the sphere of international collaboration, the Weizmann Institute maintains strong-and in many cases, formal-ties with preeminent research institutions throughout the world. The Institute attracts many foreign scientists (about 600 a year work in its laboratories for varying periods of time) and the Rehovot campus has become a popular venue for international scientific conferences, attracting over a dozen such gatherings each year.

The Weizmann Institute's relations with European institutions, including collaborative projects and the exchange of postdoctoral fellows, have recently been bolstered by the signing of an agreement that fully associates Israel with the European Union's research and development activities. To facilitate the burgeoning collaborations with European academic and industrial bodies, the Institute recently established a Liaison Office to the European Union in Brussels.

A showcase of Israel's potential, the Weizmann Institute continues to set new standards for Israel, while internationally, the Institute's reputation helps forge vital links that are absolutely essential for the nation's progress. 